\title{
Role of Perioperative TEE in Diagnosing and Management of a Case of Dynamic LVOT Obstruction/SAM
}

\author{
${ }^{1}$ Alok Kumar, ${ }^{2}$ V Rajkumar, ${ }^{3}$ Ajay Kumar, ${ }^{4} \mathrm{GD}$ Puri
}

\begin{abstract}
Systolic anterior motion (SAM) of the mitral leaflets can lead to hemodynamic instability in post bypass period. Perioperative transesophageal echocardiography (TEE) plays a crucial role in the management of SAM. Perioperative echocardiography helps to (a) identify cause of left ventricular outflow tract obstruction, (b) assess SAM and diagnose severity and (c) management of SAM. A case is presented illustrating identification and management of SAM in post bypass period.
\end{abstract}

Keywords: LVOT obstruction, Perioperative TEE, SAM.

How to cite this article: Kumar A, Rajkumar V, Kumar A, Puri GD. Role of Perioperative TEE in Diagnosing and Management of a Case of Dynamic LVOT Obstruction/SAM. J Perioper Echocardiogr 2015;3(2):58-61.

Source of support: Nil

Conflict of interest: None

\section{INTRODUCTION}

Systolic anterior motion (SAM) of the mitral valve is known to be a cause of left ventricular outflow tract (LVOT) obstruction, and is usually associated with hypertrophic cardiomyopathy (HCM) or a complication of mitral valve repair (MVR). Systolic anterior motion either transient (hemodynamic) or permanent (anatomic) may become a cause of hypotension in post bypass period and is sometimes treated by fluids, vasopressors and negative inotropic agents, such as b-blockers. Perioperative transesophageal echocardiography (TEE) plays a crucial role in the management of SAM. We report a case of LVOT obstruction by SAM of the mitral valve, who underwent coronary artery bypass graft (CABG), which was managed perioperatively conservatively.

\footnotetext{
${ }^{1,2}$ Senior Resident (Cardiac Anesthesia), ${ }^{3}$ Consultant, ${ }^{4}$ Professor

1,2,4 Department of Anesthesia and Intensive Care, Postgraduate Institute of Medical Education and Research, Chandigarh, India

${ }^{3}$ Department of Anesthesia and Intensive Care, Medanta Gurgaon, Haryana, India
}

Corresponding Author: Alok Kumar, Senior Resident (Cardiac Anesthesia), Department of Anesthesia and Intensive Care Postgraduate Institute of Medical Education and Research Chandigarh-160012, India, Phone: 08146044104, e-mail: docsomi@yahoo.com

\section{CASE REPORT}

Sixty years old male patient diagnosed with coronary artery disease (triple vessel disease), with normal left ventricle systolic function presented with history of acute onset chest pain 6 months back when he was managed medically. Since then patient had intermittent chest pain on exertion (angina on Exertion-III) and increasing dyspnea (dyspnea on Exertion-III). Patient was diagnosed as hypertensive since last 6 months and controlled on medication. On examination pulse rate of 78 per minute, systemic blood pressure of 110/70 mm Hg and an end diastolic murmur grade III at aortic area was found. Twodimensional transthoracic echocardiography (2D TTE) showed sclerodegenerative aortic valve, moderate Aortic stenosis, maximum pressure gradient $-50 \mathrm{~mm} \mathrm{Hg}$ (mean$20 \mathrm{~mm} \mathrm{Hg}$ ), aortic forward velocity-3.0 m/s, mild aortic regurgitation, concentric left ventricular hypertrophy with normal left ventricle systolic function (LV ejection fraction 55-60\%), LV diastolic dysfunction grade I and no regional wall motion abnormality (RWMA). Cardiac catheterization study revealed triple vessel disease with left main vessel being normal.

Patient was taken up for CABG and aortic valve replacement (AVR). Anesthetic management included an opioid-based, volatile anesthetic with muscle relaxants. Invasive hemodynamic monitoring and TEE was done using 2D TEE probe (6VT-D probe of the GE vivid E9 echocardiography system: GE Medical Systems, Horten, Norway). Preoperative TEE revealed sclerodegenerative aortic valve, no AS and AR, turbulence was seen in LVOT. On examination revealed a maximum velocity of $2.2 \mathrm{~m} /$ sec and gradient of $18 \mathrm{~mm} \mathrm{Hg}$ in LVOT. Left ventricular wall hypertrophy of $12 \mathrm{~mm}$ (septum) and $18 \mathrm{~mm}$ (posterior wall) was noted. Anterior mitral leaflet was $23 \mathrm{~cm}$ and PML was $19 \mathrm{~mm}, \mathrm{C}$ sept distance was $12 \mathrm{~mm}$. Decision of AVR was abandoned and only CABG was performed under cardiopulmonary bypass (CPB).

On coming off CPB patient had sustained hypotension. Left ventricular outflow tract obstruction was seen with a maximum velocity of $4 \mathrm{~m} / \mathrm{sec}$ and pressure gradient of $65 \mathrm{~mm} \mathrm{Hg}$ (Figs 1 and 2). A posteriorly directed jet of magnetic resonance (MR) was also visualized simultaneously (Fig. 3). It responded to fluid administration and vasopressors, and no further intervention 


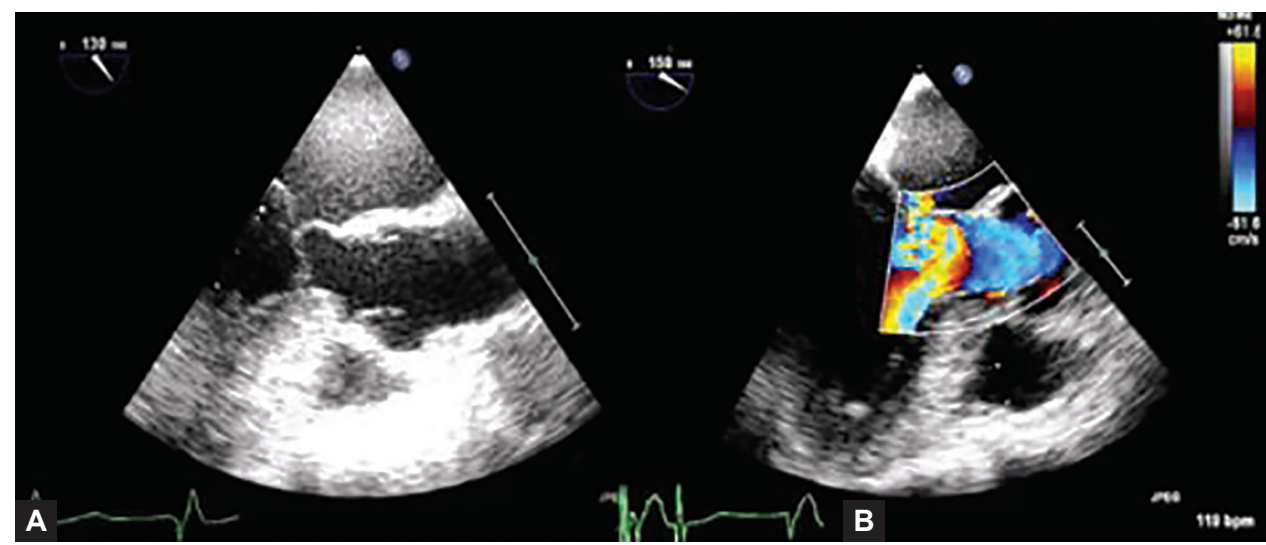

Figs 1A and B: (A) ME AV long axis view showing AML leaflet obstructing LVOT during systole, (B) turbulence during systole in LVOT (ME: Mid esophageal; AV: Aortic valve; AML: Anterior mitral leaflet; LVOT: Left ventricular outflow tract)

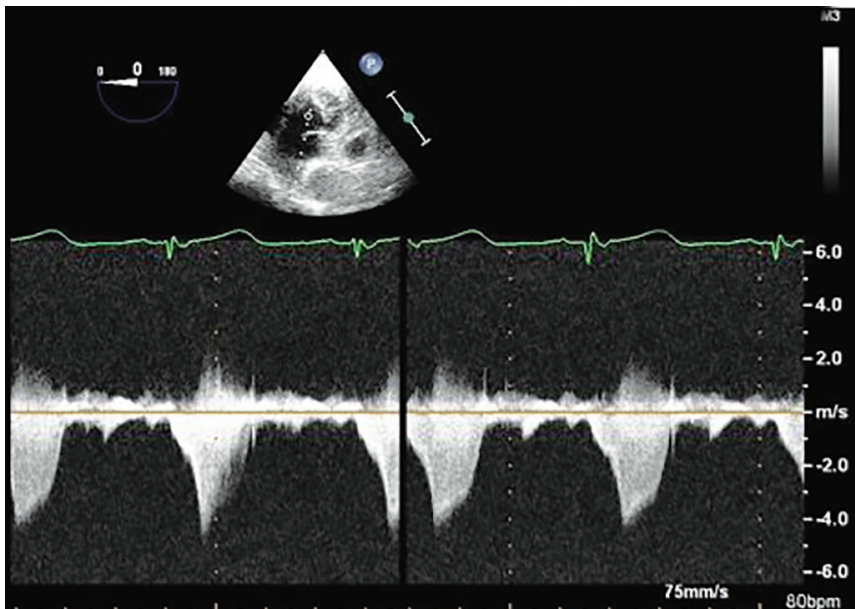

Fig. 2: Continuous wave Doppler recording in dynamic LVOT obstruction in deep transgastric view. Note the dagger-shaped profile (LVOT: Left ventricular outflow tract)

was required. Nor adrenalin $0.1 \mathrm{mcg} / \mathrm{kg} / \mathrm{min}$ was used to support the hemodynamics to achieve the perioperative goals. The patient was successfully weaned from the ventilator and discharged after an uneventful recovery.

\section{DISCUSSION}

Dynamic LVOT obstruction (LVOTO) is classically described in HCM. However, it must be appreciated that not all dynamic LVOTO is due to the former. ${ }^{1}$ If ventricular systolic function becomes hyperdynamic in a patient with basal septal hypertrophy, the LVOT becomes obstructed in a dynamic fashion, and the hemodynamic profile is similar to that observed in hypertrophic obstructive cardiomyopathy. This is most often seen in elderly patients with a clinical history of hypertension; however, the latter is not a prerequisite. ${ }^{2}$

There is agreement that SAM is caused by the action of left ventricular (LV) flow on the protruding mitral valve leaflet. The nature of the hemodynamic force on the leaflet remains a subject of unresolved debate. Initially, investigators hypothesized that anterior motion is caused by a Venturi mechanism, whereby high velocity flow in

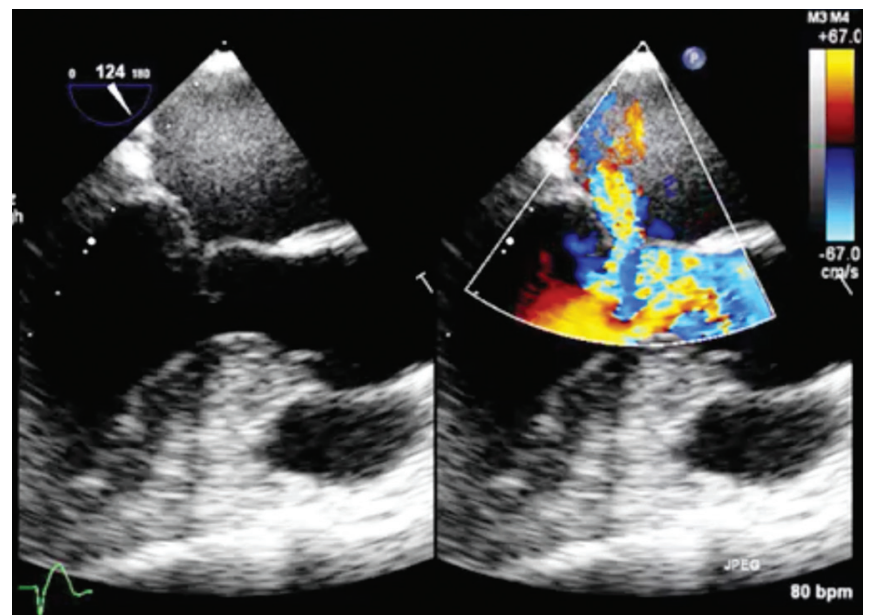

Fig. 3: ME AV long-axis view depicting LVOT turbulence and posteriorly directing jet of MR (ME: Mid esophageal; AV: Aortic valve; LVOT: Left ventricular outflow tract)

the outflow tract lifts the mitral valve toward the septum. More recent investigations indicate that drag, the pushing force of flow, is the dominant hydrodynamic force that initiates anterior motion, pushing the protruding mitral leaflet into the septum. ${ }^{3}$ Flow drag is the component of force on a body, i.e. in the direction of the flow-examples are the familiar pushing force of rushing water or the wind. The drag forces that create SAM play an important role in the generation of an LVOT gradient. The extent of septal hypertrophy and resultant narrowing of the LVOT also contribute to the LVOT gradient. ${ }^{4}$

Studies have related SAM to abnormalities of the mitral valve apparatus itself. Contributing factors include the position of papillary muscles and mitral leaflet anatomy. Anteriorly displaced papillary muscles and malposition the mitral valve toward the left ventricular outflow tract increases the likelihood of SAM and LVOTO. In particular, a relatively large posterior leaflet (PL) may coapt with the anterior leaflet (AL) closer to its base and cause both an anterior shift of the coaptation point, and an increase in the amount of slack leaflet tissue in the outflow tract. The residual leaflet portion beyond the 


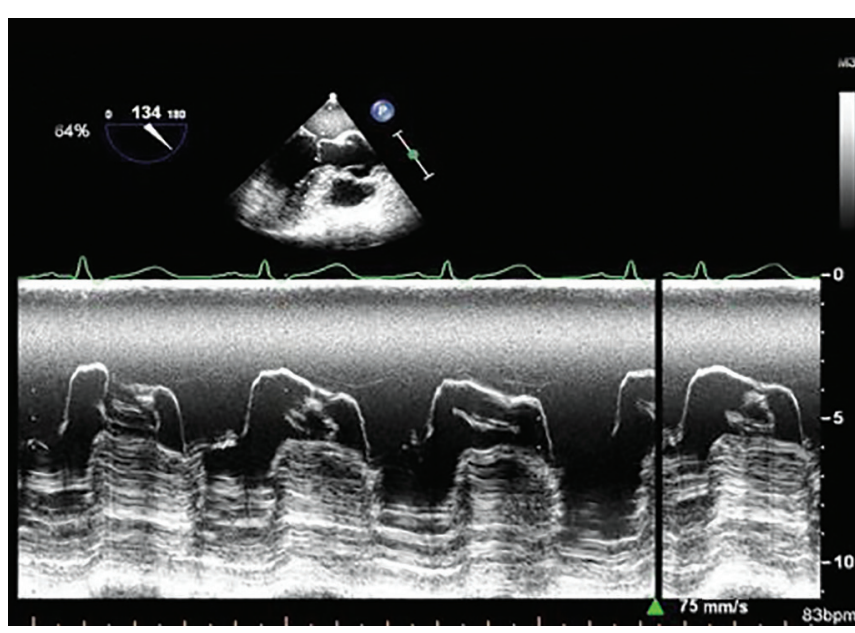

Fig. 4: M-mode across the LVOT depicting mild systolic anterior motion with septal contact of AML obstructing the LVOT in ME AV long-axis view (ME: Mid esophageal; AV: Aortic valve; AML: Anterior mitral leaflet; LVOT: Left ventricular outflow tract)

coaptation point, unlike the coapted portions that are held in place by the transmitral pressure difference, are relatively free to move in response to flow-related forces in the LVO. Elongation of the anterior leaflet may cause a similar increase in the relatively slack residual leaflet. Both of these conditions make the leaflets more susceptible to the effects of systolic outflow. ${ }^{5}$ Simple precardiopulmonary bypass mitral valve measurements, as presented above, may help to guide surgical repair and prevent SAM/LVOTO but not always. While the length of the PL and position of coaptation are important targets, the length of the anterior leaflet, the AL/PL ratio $(<1.0)$, $\mathrm{C}$-sept distance (coaptation point to the most prominent part of the septum $<2.5 \mathrm{~cm}$ ) and perhaps the amount of tissue beyond the coaptation point are important as well. ${ }^{6}$ The findings are consistent with the concept that SAM of mitral leaflets is due to anterior malposition of slack mitral leaflet portions into the LVOT which was present in our case (Fig. 4).

Also SAM nearly always results in failure of normal leaflet coaptation and mitral regurgitation (MR), which is typically mid-to-late systolic and inferolaterally oriented. SAM-related MR is inherently dynamic in nature and its severity varies with the degree of LVOTO. The presence of a central- or anteriorly directed jet of MR should raise suspicion of an intrinsic mitral valve abnormality and prompt further assessment with TEE if necessary. ${ }^{7}$

The degree of LVOTO is readily assessed with continuous wave Doppler echocardiography. Increased flow velocity across the LVOT is optimally detected from the transgastric views [(deep transgastric view or transgastric long-axis (LAX) view)] in two 2D TEE. Align the pulsed wave (PW) cursor through the aortic valve. Place the sample volume in the LVOT until a smooth wave with minimal spectral broadening is seen.
The resulting Doppler spectrum has a characteristic latepeaking dagger-shaped appearance (Fig. 2). The peak velocity detected can be used to calculate the maximum instantaneous pressure gradient across the LVOT. By convention, LVOTO is defined as an instantaneous peak Doppler LV outflow tract pressure gradient $\geq 30 \mathrm{~mm} \mathrm{Hg}$ at rest or during physiological provocation, such as Valsalva maneuver, standing and exercise. A gradient of $\geq 50 \mathrm{~mm} \mathrm{Hg}$ is usually considered to be the threshold at which LVOTO becomes hemodynamically important. When a gradient is detected in the LV cavity, it is important to systematically exclude obstruction that is unrelated to SAM, including subaortic membranes, mitral valve leaflet abnormalities and mid-cavity obstruction, particularly when interventions to relieve LV outflow obstruction are contemplated. Systematic 2D and Doppler echocardiography is usually sufficient to determine the mechanism and severity of LVOTO. ${ }^{7}$

Approximately, one-third of patients have resting SAM of the mitral valve leaflets that results in obstruction to the LV outflow tract, while another third have latent obstruction only during maneuvers that change loading conditions and LV contractility. There are specialized situations, such as in the operating room or intensive care unit, in which the pathophysiologic settings can mimic obstructive HCM. An example of this is the postoperative patient with basal septal hypertrophy or sigmoid septum, in which the left ventricle is underfilled coming off bypass. In this situation, a number of factors converge and produce SAM along with LVOT obstruction. These include elongated mitral leaflets, a narrow LVOT, a small LV cavity, use of inotropes, hypervolemia and hyperdynamic EF.8 In general, these can be reversed with volume loading, afterload increase, and stopping inotropic agents. A simple two-step conservative management (expanding intravascular volume and discontinuing any inotropic drug as a first step and, most importantly, increasing the afterload through vasopressors as a second step) resolves the SAM in most patients and allowed to identify those few patients who needed surgical revision. The main problem that surgeons and anesthesiologists face in the theater is how to differentiate between transient (hemodynamic) and permanent (anatomic) SAM. While 'hemodynamic SAM' can be solved by reducing the tachycardia, the anatomic SAM' needs surgical repair. It is likely that patients with postoperative SAM subsequently have recurring episodes of catecholamine surges as well as intermittent hypovolemia. During these episodes, these patients have demonstrated the anatomic architecture to develop SAM and significant MR. Repeated episodes of this may be the underlying pathology that results in a worse outcome in these patients as suggested by an observational study. ${ }^{10}$ 
Systolic anterior motion is a frequent cause of LVOT obstruction in post bypass period. Arising from the mismatch between size and position of the mitral leaflets and hypertrophied septum, SAM can clinically cause significant hemodynamic compromise postoperatively. Initial medical management includes increasing preload and maintaining adequate afterload with avoidance of inotropes. However, reoperations involving repair/ replacement of valve or septal ablation may be required in cases of severe SAM.

\section{REFERENCES}

1. Gupta R, Sewani A, Ahmad M. Dynamic systolic left ventricular gradients: differential diagnosis and management. Echocardiography 2006;23(2):168-171.

2. Topol EJ, Traill TA, Fortuin NJ. Hypertensive hypertrophic cardiomyopathy of the elderly. N Engl J Med 1985;312(5): 277-283.

3. Sherrid MV, Gunsburg DZ, Moldenhauer S, Pearle G. Systolic anterior motion begins at low left ventricular outflow tract velocity in obstructive hypertrophic cardiomyopathy. J Am Coll Cardiol 2000;36(4):1344-1354.

4. Gersh BJ, Maron BJ, Bonow RO, et al. ACCF/AHA Guideline for the diagnosis and treatment of Hypertrophic Cardiomyopathy: a report of the American College of Cardiology Foundation/ American Heart Association Task Force on Practice Guidelines
Developed in Collaboration with the American Association for Thoracic Surgery, American Society of Echocardiography, American Society of Nuclear Cardiology, Heart Failure Society of America, Heart Rhythm Society, Society for Cardiovascular Angiography and Interventions, and Society of Thoracic Surgeons. J Am Coll Cardiol 2011;58(25):e212-e260.

5. Maslow AD, Regan MM, Haering JM, Johnson RG, Levine RA. Echocardiographic predictors of left ventricular outflow tract obstruction and systolic anterior motion of the mitral valve after mitral valve reconstruction for myxomatous valve disease. J Am Coll Cardiol 1999;34(7):2096-2104.

6. Landoni G, Crescenzi G, Zangrillo A, Nicolotti D, Bignami E, Iaci G, Alfieri O, Guarracino F. Validation of a decisionmaking strategy for systolic anterior motion following mitral valve repair. Ann Cardiac Anaesth 2011;14(2):85-90.

7. Elliott PM, Anastasakis A, Borger MA, et al. ESC Guidelines on diagnosis and management of hypertrophic cardiomyopathy. Eur Heart J 2014;35:2733-2779.

8. YangJH, Park SW, Yang JH, ChoSW, Kim HS, Choi KA, Kim HJ. Dynamic left ventricular outflow tract obstruction without basal septal hypertrophy, caused by catecholamine therapy and volume depletion. Korean J Int Med 2008;23:106-109.

9. Auer J, Berent R, Weber T, Lamm G, Eber B. Catecholamine therapy inducing dynamic left ventricular outflow tract obstruction. Int J Cardiol 2005;101:325-328.

10. Sorrell VL, Habibzadeh MR, Kalra N, Chitwood WR. Transient severe mitral regurgitation after mitral valve repair is associated with a poor clinical outcome: a small case series. J compilation. Echocardiography 2008;25:835-839. 